\title{
The interaction between pro-inflammatory cytokines and the nervous system
}

\author{
Alessio Molfino, Filippo Rossi-Fanelli and Alessandro Laviano
}

As recently reviewed by Seruga et al. ${ }^{1}$ (Cytokines and their relationship to the symptoms and outcome of cancer. Nature Rev. Cancer 8, 887-899; 2008), pro-inflammatory cytokines modulate a number of biochemical and behavioural responses that contribute to the clinical characterization of acute and chronic diseases. Therefore, better understanding of the mechanisms of action of cytokines may help in devising effective therapeutic strategies to counteract their systemic effects, which affect patients' quality of life and ultimately their outcome.

Pro-inflammatory cytokines are involved in the development of cancer-associated anorexia and cachexia, and are more common in certain types of cancer ${ }^{1}$. We agree with Seruga et al. that a number of studies indicate that cytokines induce muscle wasting by promoting the proteolysis of skeletal muscle and the uncoupling of mitochondrial respiration ${ }^{2,3}$ and suggest that these effects are locally mediated. However, as correctly pointed out by Seruga et al., the use of etanercept as an anti-cachexia agent failed to yield significant clinical benefits ${ }^{4}$, suggesting that pro-inflammatory cytokines may promote wasting during cancer by mechanisms that do not only involve their effects on peripheral tissues.

Recent evidence indicate that energy homeostasis, that is, the control of energy intake and expenditure, is largely mediated in the hypothalamus, and centrally produced cytokines are involved in triggering the molecular changes associated with the development of cancer associated anorexia and cachexia ${ }^{5}$. Also, experimental models of wasting indicate that muscle proteolysis during disease is influenced by hypothalamic activity. In particular, centrally produced pro-inflammatory cytokines appear to divert the activity of the hypothalamic melanocortin system largely toward the promotion of catabolic stimuli.

The interaction between proinflammatory cytokines and the nervous system may occur at the peripheral levels and may represent one of the first molecular events triggering the host inflammatory response. The host inflammatory response, when it is chronically persistent, may lead in turn to the development of anorexia and cachexia. Indeed, tumour growth could be sensed by the vagus nerve, possibly through local interaction with pro-inflammatory cytokines. This information is conducted to brainstem areas and then to the hypothalamus, yielding to the activation of the melanocortin system through cholinergic, serotonergic and noradrenergic innervation. The melanocortin system, when activated, stimulates the synthesis of pro-inflammatory cytokines to maintain the catabolic state. Supporting this view, hypothalamic neurons regulating energy homeostasis express the type 1 interleukin 1 (IL-1) receptor, and intracerebroventricular injection of IL-1 increases their activity ${ }^{6}$. Finally, the activation of the melanocortin system also induces metabolic changes on peripheral tissues, at least in part, through sympathetic outflow.
The role of pro-inflammatory cytokines, and in particular IL-1 and tumour necrosis factor, in the pathogenesis of anorexia and cachexia has been recognized for many years ${ }^{7}$. More recently, it has been shown that they may be involved in mediating the onset of the host inflammatory response to tumour growth through interaction with the peripheral nervous system at the peripheral level ${ }^{8}$, and that their hyperexpression in the central nervous system may sustain the metabolic and behavioural changes observed in cancer patients ${ }^{9}$.

Consequently, inhibition of the central inflammatory response that is induced by cytokines may result in better clinical outcome than systemic immune suppression.

Alessio Molfino, Filippo Rossi-Fanelli and Alessandro Laviano are at the Department of Clinical Medicine, Sapienza University, Rome, Italy. Correspondence to A.L. e-mail: alessandro.laviano@uniroma1.it Published online 5 February 2009

1. Seruga, B., Zhang, H., Bernstein, L. J. \& Tannock, I. F. Cytokines and their relationship to the symptoms and outcome of cancer. Nature Rev. Cancer 8, 887-899 (2008).

2. Bossola, M. et al. Increased muscle proteasome activity correlates with disease severity in gastric cancer patients: Ann. Surg. 237, 384-389 (2003).

3. Busquets, S. et al. Tumour necrosis factor-a uncouples respiration in isolated rat mitochondria. Cytokine $\mathbf{2 2}$, 1-4 (2003).

4. Jatoi, A. et al. A placebo-controlled double blind trial of etanercept for the cancer anorexia/weight loss syndrome: results from $\mathrm{NOOC} 1$ from the North Central Cancer Treatment Group. Cancer 110, 1396-1403 (2007).

5. Laviano, A. et al. Neural control of the anorexiacachexia syndrome. Am. J. Physiol. Endocrinol. Metab. 295, 1000-1008 (2008).

6. Scarlett, J. M. et al. Regulation of central melanocortin signalling by interleukin- $1 \beta$. Endocrinology 148, 4217-4225 (2007).

7. Inui, A. Cancer anorexia-cachexia syndrome: are neuropeptides the key? Cancer Res. 59, 4493-4501 (1999).

8. Ruud, J. \& Blomqvist, A. Identification of rat brainstem neuronal structures activated during cancer-induced anorexia. J. Comp. Neurol. 504, 275-286 (2007).

9. Laviano, A., Meguid, M. M. \& Rossi Fanelli, F. Cancer anorexia: clinical implications, pathogenesis and therapeutic strategies. Lancet Oncol. 4, 686-694 (2003). 